\title{
Influência do período de alimentação no consumo e ganho de peso do pirarucu
}

\author{
Roger Crescêncio(1), Daniel Rabello Ituassú(2), Rodrigo Roubach(2), Manoel Pereira Filho(2), \\ Bruno Adan Sagratzki Cavero ${ }^{(3)}$ e André Lima Gandra ${ }^{(2)}$
}

\begin{abstract}
(1)Embrapa Acre, Caixa Postal 321, CEP 69908-970 Rio Branco, AC. E-mail: roger@cpafac.embrapa.br (2)Instituto Nacional de Pesquisas da Amazônia, Coordenação de Pesquisas em Aqüicultura, Caixa Postal 478, CEP 69083-000 Manaus, AM. E-mail: dituassu@inpa.gov.br, roubach@inpa.gov.br, pmanoel@inpa.gov.br, andrelimagandra@yahoo.com.br (3)Universidade Federal do Amazonas, Fac. de Ciências Agrárias, Dep. de Ciências Pesqueiras, Av. Gen. Rodrigo Otávio Jordão Ramos, no 3.000, Coroado, CEP 69077-000 Manaus, AM. E-mail: basc@ufam.edu.br
\end{abstract}

Resumo - O objetivo deste trabalho foi verificar o período preferencial de consumo alimentar do pirarucu, Arapaima gigas, e a influência de diferentes turnos de alimentação no consumo e ganho de peso dessa espécie. Foram testados três tratamentos: alimentação diurna (peixes alimentados às $9 \mathrm{~h}$ e 15h), alimentação noturna (peixes alimentados às $21 \mathrm{~h}$ e $3 \mathrm{~h}$ ) e alimentação contínua (peixes alimentados às 9h, 15h, 21h e 3h). Cada tratamento foi avaliado em triplicata, com cada unidade experimental formada por oito peixes, com peso médio de $313 \mathrm{~g}$, estocados em tanques-redes de $1 \mathrm{~m}^{3}(1 \mathrm{x} 1 \mathrm{x} 1 \mathrm{~m})$. Os nove tanques-redes foram alocados em um viveiro escavado de $120 \mathrm{~m}^{2}$. O experimento durou 60 dias. A alimentação contínua promoveu maiores ganhos de peso e biomassa, taxa de crescimento específico e consumo total. Os tratamentos alimentação diurna e alimentação noturna apresentaram ganho de peso semelhante, porém, a alimentação diurna apresentou a melhor conversão alimentar. O período preferencial de alimentação do pirarucu foi o noturno, principalmente no começo da noite. Os dados deste estudo indicam que o horário de preferência alimentar não é o melhor horário de alimentação do pirarucu, em uma criação comercial. O período de alimentação mais indicado para a espécie, baseado na capacidade de conversão alimentar, é o diurno.

Termos para indexação: Arapaima gigas, conversão alimentar, manejo alimentar.

\section{Influence of feeding period on consumption and weight gain of pirarucu}

\begin{abstract}
The objective of this study was to verify pirarucu Arapaima gigas preferential time of feeding, and the influence of different feeding periods on consumption and weight gain of this species. Three treatments were tested: diurnal feeding (fish fed at $9 \mathrm{~h}$ and $15 \mathrm{~h}$ ), nocturnal feeding (fish fed at $21 \mathrm{~h}$ and $3 \mathrm{~h}$ ), and continuous feeding (fish fed at 9h, 15h, 21h and 3h). Each treatment was evaluated in triplicate, and each trial unit had eight fishes with 313 g average weight, stoked into a $1 \mathrm{~m}^{3}(1 \times 1 \times 1 \mathrm{~m})$ net cage. The nine net cages were located on a $120 \mathrm{~m}^{2}$ earth pond. The trial lasted for 60 days. Continuous feeding promoted the highest weight and biomass gain, specific growth rate and feed consumption. Nocturnal and diurnal feeding treatments showed similar weight gain; however, diurnal feeding had the best feed conversion ratio. The preferred feeding time for pirarucu was the nocturnal period, mainly at the beginning of night. Data from this study indicated that the preferred feeding time is not the best one for feeding pirarucu on a commercial husbandry. The most indicated feeding period for this species, based on its feed conversion capacity, is diurnal period.
\end{abstract}

Index terms: Arapaima gigas, feed conversion ratio, feed management.

\section{Introdução}

O gasto com ração é um dos itens que mais influenciam na lucratividade da piscicultura intensiva. O emprego de manejo alimentar correto pode proporcionar uma taxa de ingestão que melhore a relação entre a quantidade de alimento fornecida e a produção de biomassa e que, conseqüentemente, diminua o gasto com ração na produção de uma mesma biomassa de peixe, ou reduza o tempo de cultivo.

A taxa de ingestão alimentar e o aproveitamento de nutrientes pelos peixes estão diretamente relacionados ao horário de alimentação (Boujard \& Leatherland, 1992). Durante o ciclo circadiano, os peixes apresentam variações: na intensidade de procura por alimento (Boujard, 1995); na segurança de não encontrar preda- 
dores (Greenwood \& Metcalfe, 1998); nos picos de produção de enzimas digestivas (López-Vásquez, 2001); da digestibilidade e da síntese de proteínas para formação do tecido muscular (Gelineau et al., 1996; Bolliet et al., 2000). Essas variações circadianas, ligadas à alimentação, dependem também da espécie, da fase da vida, da fase do ciclo anual e do convívio intra e interespecífico (Boujard, 1995; Greenwood \& Metcalfe, 1998; Jobling et al., 1998; Amundsen et al., 2000; Imre \& Boiscalar, 2004).

Diversos experimentos foram realizados, para identificar o manejo alimentar adequado para espécies de importância econômica, tendo-se buscado empregar, no cultivo, o padrão alimentar apresentado pelos peixes na natureza. Peixes como a pirapitinga, Piaractus brachypomus, a perca, Dicentrarchus labrax, e o bagre europeu, Silurus glanis, apresentam melhores ganhos de peso e conversões alimentares, quando alimentados em seus horários preferenciais de alimentação na natureza, no período noturno (Boujard, 1995; Baras et al., 1996; Azzaydi et al., 2000). Entretanto, em estações do ano nas quais a perca apresenta preferência diurna, a aplicação de seu padrão natural diurno, no cultivo, acelera seu ganho de peso e melhora sua conversão alimentar (Azzaydi et al., 1999).

O pirarucu (A. gigas) é um peixe que vem gerando grandes expectativas em torno de sua criação, em razão da qualidade e preço de sua carne e de seu crescimento rápido. Ele pode atingir $10 \mathrm{~kg}$ de peso médio, ao final de um ano (Imbiriba, 2001), e sua produção estimada é de $25 \mathrm{t} \mathrm{ha}^{-1}$ por ano, quando alimentado com ração em sistema de criação intensiva (Pereira-Filho et al., 2003). Essa espécie apresenta respiração aérea obrigatória, possibilitada por sua bexiga natatória modificada, que permite a tomada de oxigênio diretamente do ar atmosférico. Por não necessitar do oxigênio dissolvido na água, suporta elevadas densidades de estocagem em cultivo, por isso pode aumentar a produção por área e ser cultivado em locais não propícios a outras espécies aquáticas (Ono et al., 2004).

Gandra (2002), ao estudar a freqüência alimentar do pirarucu, sugeriu a alimentação duas vezes ao dia, como a melhor estratégia para a criação dessa espécie. Porém, pouco se conhece a respeito da preferência alimentar natural do pirarucu, quanto ao horário de alimentação, e sobre o efeito que diferentes manejos alimentares podem causar no desempenho zootécnico dessa espécie.
O objetivo deste trabalho foi identificar o horário preferencial de alimentação do pirarucu, bem como o efeito de diferentes turnos alimentares (diurno, noturno e contínuo) no consumo de alimento e no ganho de peso dessa espécie.

\section{Material e Métodos}

O experimento foi realizado nas dependências da Coordenação de Pesquisas em Aqüicultura (CPAQ), do Instituto Nacional de Pesquisas da Amazônia (Inpa), de 17 de setembro a 16 de novembro de 2001. Foram avaliados três tratamentos: alimentação diurna (AD), em que os peixes foram alimentados duas vezes por dia, durante o período diurno (9h e 15h); alimentação noturna (AN), em que os peixes foram alimentados duas vezes por dia, durante o período noturno (21h e 3h); e alimentação contínua (AC), em que os peixes foram alimentados quatro vezes por dia, durante os períodos diurno e noturno (9h, 15h, 21h e 3h). Cada tratamento foi testado em triplicata. A unidade experimental foi constituída de oito juvenis de pirarucu, com peso médio de $318 \pm 23$ g, $301 \pm 16$ g e $319 \pm 27$ g para os tratamentos AD, AC e AN, respectivamente. Os peixes foram mantidos em um tanque-rede de $1 \mathrm{~m}^{3}$ (1x1x1 m), num total de nove tanques-redes com 72 juvenis e uma biomassa inicial de $2.504 \mathrm{~g}$ por tanque-rede. Os tanques-redes foram alojados em um tanque escavado de $120 \mathrm{~m}^{2}$.

Os peixes foram alimentados, até a saciação aparente, com uma ração comercial extrusada para peixes carnívoros, com $40 \%$ de proteína bruta. Durante a alimentação noturna, o consumo do alimento foi observado com auxílio de uma lanterna. Cada unidade experimental possuía um pote de ração respectivo, mantido em laboratório até o momento do arraçoamento. Os potes de ração eram pesados antes e depois de cada alimentação, e por diferença foi obtido o total de ração fornecido por tanque e por horário de alimentação.

Foram realizadas cinco biometrias, uma inicial e uma a cada 15 dias de experimento, que tiveram a duração de 60 dias. Em dias de biometria, feitas durante o período diurno, os peixes não foram alimentados em nenhum dos horários. Foram analisados os seguintes parâmetros de desempenho: ganho de peso ( $\mathrm{g}$ ) = peso médio final ( $\mathrm{g}$ ) - peso médio inicial (g); ganho de biomassa (g) = biomassa final (g) - biomassa inicial (g); consumo total (g); conversão alimentar aparente = consumo (g)/ganho de peso (g); taxa de crescimento específico (\%) = $100 \mathrm{x}$ (Ln biomassa final - Ln biomassa inicial) x número de dias. Para se 
conhecer a preferência da espécie, relativa ao horário de alimentação, foi avaliado o consumo de ração total por horário, de cada tratamento separadamente. Nessa avaliação, não foram analisados os dados dos dois dias posteriores a cada biometria, pois ocorreu uma tendência à hiperalimentação no primeiro arraçoamento do dia, o que poderia mascarar a preferência real dos peixes.

A água do tanque foi renovada para repor as perdas por evaporação. A qualidade da água foi avaliada, diariamente, quanto ao $\mathrm{pH}$, temperatura $\left({ }^{\circ} \mathrm{C}\right)$ e condutividade $\left(\mu \mathrm{Sm} \mathrm{cm}^{-1}\right)$, e a cada cinco dias foi verificado o teor de nitrito $\left(\mathrm{mg} \mathrm{L}^{-1}\right)$. A temperatura média da água foi $29,3 \pm 0,8^{\circ} \mathrm{C}$, o $\mathrm{pH}$ médio verificado foi $7,02 \pm 0,33$, a condutividade média foi 77,4 $\pm 21,1 \mu \mathrm{Sm} \mathrm{cm}^{-1}$ e o nível médio de nitrito foi de $0,14 \pm 0,14 \mathrm{mg} \mathrm{L}^{-1}$.

Os dados referentes a consumo total, ganho de peso, ganho de biomassa, conversão alimentar aparente e taxa de crescimento específico foram analisados por ANOVA one-way, e quando identificadas diferenças significativas, as médias foram discriminadas pelo teste de Duncan $(\mathrm{p}<0,05)$. Os dados de consumo por horário, referentes ao tratamento com alimentação contínua, foram analisados da mesma forma que os descritos anteriormente, e os dados de consumo por horário, dos tratamentos somente com alimentação diurna ou noturna foram analisados pelo teste $t$.

\section{Resultados e Discussão}

O pirarucu na fase juvenil mostrou diferenças quanto à preferência do horário alimentar. O consumo por horário foi diferente em todos os tratamentos. No tratamento AD, o horário identificado como o de maior preferência alimentar foi às $15 \mathrm{~h}$, quando foi consumido 56,6\% do total de ração. O horário preferencial de alimentação dos peixes AN foi às 21h, quando foi consumido $60,7 \%$ do total de ração. A preferência alimentar geral dos juvenis de pirarucu ocorre no período noturno pois, nesse período, os peixes do tratamento AC consumiram 65,9\% do total diário de alimento oferecido. Ainda no tratamento AC, o horário de maior consumo foi às $21 \mathrm{~h}$ (38,6\%), e o segundo maior $(27,3 \%)$ foi às 3 h; o consumo por horário foi diferente entre todos os horários desse tratamento (Figura 1). Não houve mortalidade em nenhum dos tratamentos.

Pequenas variações diárias quanto aos horários de maior consumo foram verificadas, porém, a maioria corrobora a preferência pela noite. Uma forte indicação desse padrão noturno vem de Smith (1979), que descreve as duas principais formas de captura do pirarucu: a primeira é a pesca com arpão, realizada durante o dia e a segunda é a pesca com armadilha de espera, feita normalmente com uma isca de peixe, e deixada por toda a noite.

Os efeitos das estratégias alimentares no consumo total, ganho de peso, ganho de biomassa, conversão alimentar aparente e taxa de crescimento específico estão demonstrados na Tabela 1. Ao final do experimento, todos os tratamentos apresentaram diferenças referentes ao consumo alimentar. O consumo no tratamento $\mathrm{AD}$ foi menor que os demais até o $30^{\circ}$ dia. A partir

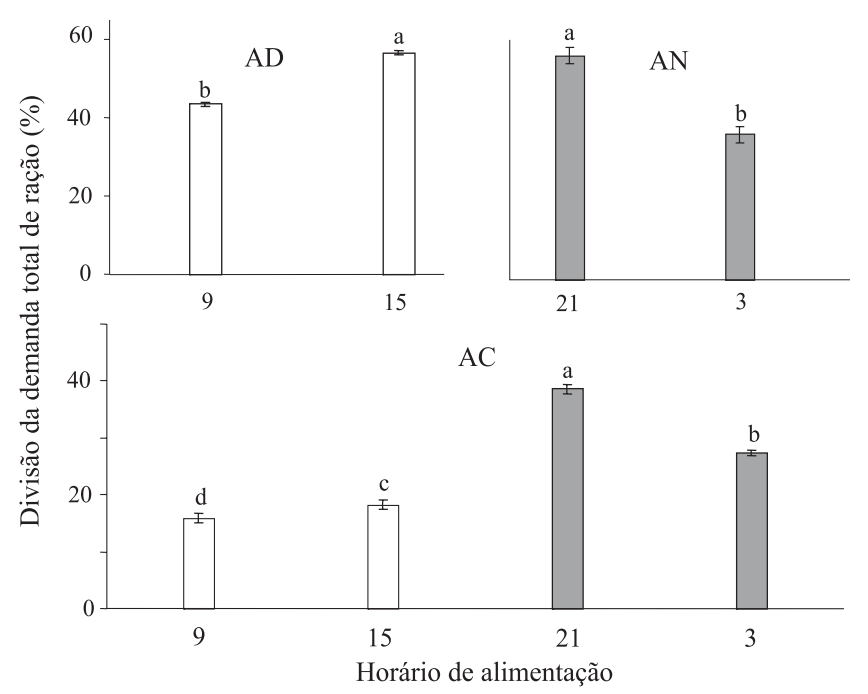

Figura 1. Consumo de ração, por horário, por juvenis de pirarucu, nos tratamentos com alimentação diurna (AD), noturna (AN) e contínua (AC). Dados expressos em média \pm desviopadrão. Valores com letras diferentes, em um mesmo tratamento, apresentam diferenças significativas com $\mathrm{p}<0,01$ (AD e AN: teste t; AC: ANOVA, teste de Duncan).

Tabela 1. Efeito da alimentação diurna (AD), noturna (AN) e contínua (AC), no consumo médio (CT), ganho de peso (GP), ganho de biomassa (GB), conversão alimentar aparente (CAA) e taxa de crescimento específico (TCE) de juvenis de pirarucu $^{(1)}$.

\begin{tabular}{lcccc}
\hline Variáveis & $\mathrm{AD}$ & $\mathrm{AN}$ & $\mathrm{AC}$ & $\mathrm{p}$ \\
\hline $\mathrm{CT}(\mathrm{g})$ & $721 \pm 68 \mathrm{c}$ & $1.065 \pm 68 \mathrm{~b}$ & $1.314 \pm 56 \mathrm{a}$ & $<0,01$ \\
$\mathrm{GP}(\mathrm{g})$ & $564 \pm 27 \mathrm{~b}$ & $567 \pm 52 \mathrm{~b}$ & $692 \pm 31 \mathrm{a}$ & $<0,01$ \\
$\mathrm{~GB}(\mathrm{~g})$ & $4.516 \pm 213 \mathrm{~b}$ & $4.539 \pm 414 \mathrm{~b}$ & $5.536 \pm 246 \mathrm{a}$ & $<0,01$ \\
$\mathrm{CAA}$ & $1,28 \pm 0,17 \mathrm{a}$ & $1,88 \pm 0,28 \mathrm{~b}$ & $1,90 \pm 0,06 \mathrm{~b}$ & $<0,05$ \\
TCE $(\%$ peso por dia) & $1,70 \pm 0,14 \mathrm{~b}$ & $1,70 \pm 0,04 \mathrm{~b}$ & $1,99 \pm 0,12 \mathrm{a}$ & $<0,05$ \\
\hline
\end{tabular}

(1)Médias seguidas da mesma letra na linha não diferem entre si pelo teste de Duncan; valores expressos em médiaさdesvio-padrão. 
do 45o dia até o fim do experimento, todos os tratamentos apresentaram diferenças significativas entre si. Ocorreu um distanciamento nas quantidades de consumo dos tratamentos; com o passar do tempo, o consumo em AC foi aumentando mais do que em AN, e o consumo em $\mathrm{AD}$ manteve-se sempre menor que nos outros dois tratamentos (Figura 2).

Os tratamentos AD e AC promoveram diferenças quanto ao ganho de peso, a partir do 30 o dia, tendo continuado com esse padrão aos 45 dias do experimento. Ao final do experimento, os tratamentos $\mathrm{AD}$ e $\mathrm{AN}$ proporcionaram ganhos de peso inferiores a AC (Figura 3). Conseqüentemente, os ganhos de biomassa apresentados pelos peixes dos tratamentos $\mathrm{AD}$ e $\mathrm{AN}$, foram inferiores aos do tratamento AC.

Ao final do experimento, a taxa de crescimento específico (TCE), proporcionada pelo tratamento AC, foi significativamente superior às do outros dois tratamentos. Até o 30 dia do experimento, os peixes alimentados somente à noite (AN) apresentaram um rápido crescimento, tendo se equiparado ao crescimento dos peixes do tratamento AC. Porém, com o passar do tempo, houve tendência de queda na TCE nos peixes de $\mathrm{AC}$ e $\mathrm{AN}$, e uma tendência de continuidade no tratamento com alimentação diurna. A primeira diferença significativa foi notada no $30^{\circ}$ dia de experimento, em que foi verificado que a TCE do tratamento AC foi superior à do tratamento $\mathrm{AD}$ (Figura 4).

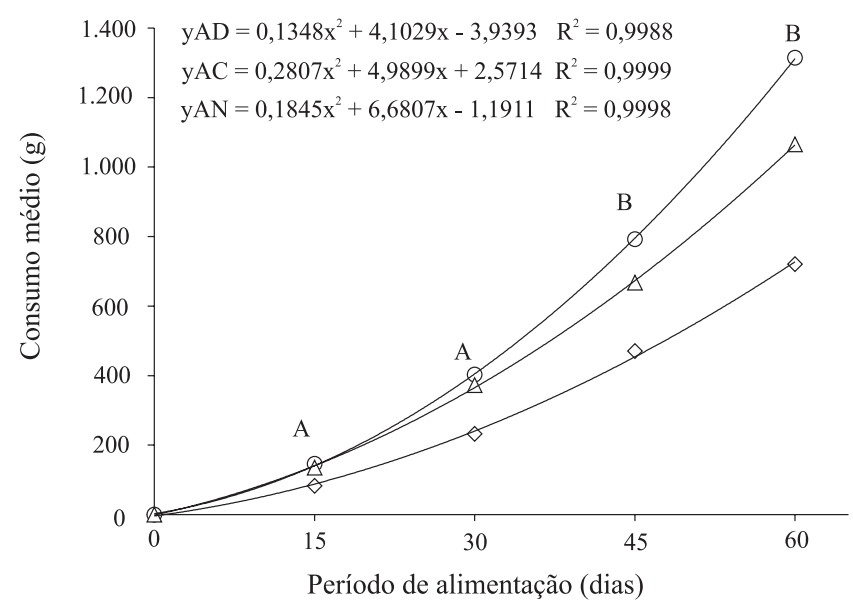

Figura 2. Consumo médio de ração por juvenis de pirarucu com alimentação diurna $(\mathrm{AD}, \diamond)$, noturna $(\mathrm{AN}, \triangle)$ e contínua (AC, $\mathrm{O}$ ), nos momentos $\mathrm{A}$, em que o tratamento $\mathrm{AD}$ é menor que os tratamentos $\mathrm{AC}$ e $\mathrm{AN}(\mathrm{p}<0,01)$, e $\mathrm{B}$, em que todos os tratamentos são diferentes entre si $(\mathrm{p}<0,01)$.
A conversão alimentar aparente (CAA) do tratamento AD foi significativamente melhor que a dos tratamentos AC e AN, e as CAA encontradas em AC e AN foram piores que as encontradas em outros experimentos, em que os pirarucus foram alimentados com ração extrusada. Em trabalho recente, juvenis de pirarucu cultivados de $0,13 \mathrm{~kg}$ até $7 \mathrm{~kg}$ de peso médio apresentaram CAA 1,5, tendo sido alimentados com a mesma ração do experimento atual, duas vezes ao dia, durante o período diurno (Pereira Filho et al., 2003). A CAA verificada no tratamento $\operatorname{AD}(1,28)$ foi próxima da encontrada em estudo com pirarucus criados de $0,04 \mathrm{~kg}$ a $1,06 \mathrm{~kg}$, em tanques-redes de $1 \mathrm{~m}^{3}$. A conversão alimentar obtida pelos autores, na época de melhor desempenho (140o dia), foi de 1,12 (Cavero et al., 2003).

Os valores de CAA dos tratamentos AC e AN foram melhores que os descritos em experimentos em que a dieta fornecida não foi a ração extrusada. Dados provenientes de pirarucus alimentados com peixes revelam conversão alimentar em torno de 4,2 e 6,5. Quando alimentado com ensilado biológico, o pirarucu apresentou CAA entre 5,9 e 35,8 (Honczaryck \& Maeda, 1998; Garcia et al., 2002). Também foi verificado que pirarucus cultivados, de $120 \mathrm{~g}$ até pesos médios finais que variaram de $231 \mathrm{~g}$ a $357 \mathrm{~g}$, alimentados com dietas peletizadas com nível de proteína bruta de 30\% a 48\%, apresentaram CAA entre 3,2 e 1,6 (Ituassú et al., 2005). Essa comparação entre dados de peixes cultivados com ração extrusada e peixes alimentados com outras dietas

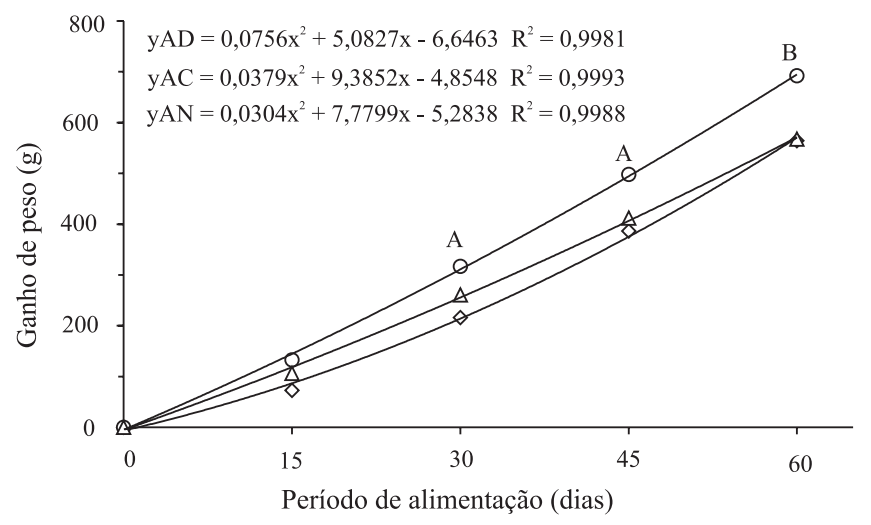

Figura 3. Ganho de peso médio de juvenis de pirarucu com alimentação diurna $(\mathrm{AD}, \diamond)$, noturna $(\mathrm{AN}, \triangle)$ e contínua (AC, $\bigcirc$ ), nos momentos $A$, em que o tratamento AD é menor que o tratamento $\mathrm{AC}(\mathrm{p}<0,05), \mathrm{B}$, em que o tratamento $\mathrm{AC}$ é superior aos tratamentos $\mathrm{AD}$ e $\mathrm{AN}(\mathrm{p}<0,05)$. 
é imprescindível, para se ressaltar a importância da utilização da ração extrusada na criação do pirarucu.

Os peixes que foram alimentados à noite - seu horário preferencial na natureza - tanto em AN quanto em AC, apresentaram maior procura por alimento e conseqüente excesso de consumo nesse período, tendo piorado a conversão alimentar. Quando alimentado no período noturno, o pirarucu comeu mais do que o necessário para o crescimento e manutenção das atividades fisiológicas. A hiperfagia noturna do pirarucu fica evidente, quando se comparam os tratamentos $\mathrm{AD}$ e AN, em que foi empregada a mesma freqüência alimentar (duas vezes ao dia). Os peixes desses tratamentos apresentaram o mesmo ganho de peso ao final do experimento, porém os peixes alimentados à noite apresentaram CAA $47 \%$ pior. Resultado semelhante foi evidenciado com o pompano da Flórida, Trachinotus carolinus, que apresentou uma conversão alimentar pior, quando alimentado em seu horário preferencial (Heilman \& Spieler, 1999).

Estudo recente demonstrou que a perca (Dicentrarchus labrax), quando alimentada à noite, perde a capacidade de capturar os grânulos de ração, aumentando o desperdício de alimento (Rubio et al., 2003). O mesmo parece não ocorrer com os pirarucus alimentados à noite, pois, neste estudo, os peixes foram alimentados com uma ração flutuante, o que permite melhor controle visual do consumo, ao contrário do experimento realizado com a $D$. labrax. O bagre africano (Clarias gariepinus) apresenta menores perdas de

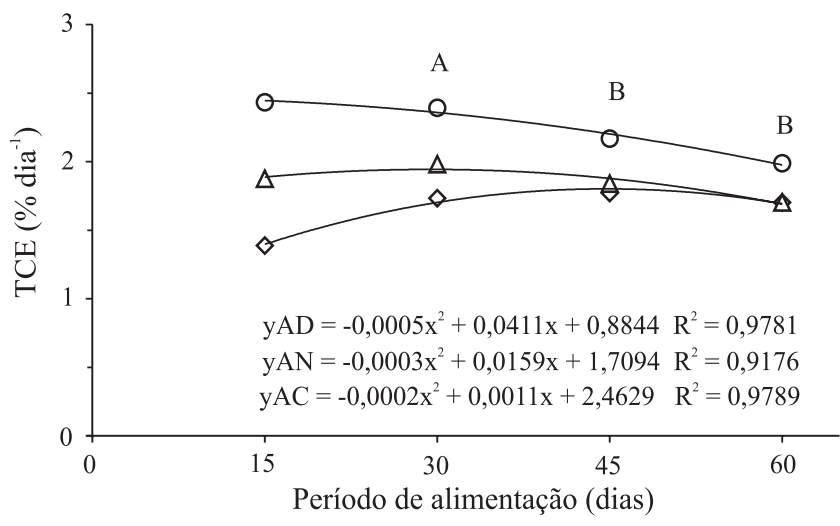

Figura 4. Taxa de crescimento específico (TCE) de juvenis de pirarucu, com alimentação diurna $(\mathrm{AD}, \diamond)$, noturna $(\mathrm{AN}, \triangle)$ e contínua (AC, $\bigcirc$ ), nos momentos $\mathrm{A}$, em que o tratamento AC é superior ao tratamento $\mathrm{AD}(\mathrm{p}<0,05)$, e $\mathrm{B}$, em que o tratamento AC é superior aos tratamentos $\mathrm{AD}$ e $\mathrm{AN}(\mathrm{p}<0,05)$. alimento e melhores ganhos de peso e conversão alimentar, quando alimentado à noite ou continuamente, do que quando alimentado durante o dia, sendo que a alimentação noturna também proporciona a melhor eficiência de utilização alimentar (Hossain et al., 2001).

Embora o tratamento AC tenha proporcionado os melhores ganhos de peso e biomassa e a maior TCE, esse tratamento promoveu aumento significativo no consumo, quando comparado ao tratamento $\mathrm{AD}$. $\mathrm{O}$ ganho de peso dos peixes alimentados continuamente foi $23 \%$ maior que o dos peixes alimentados somente no período diurno, embora o consumo tenha sido $82 \%$ maior (Tabela 1). Se o manejo alimentar de alimentação contínua fosse implantado em uma criação comercial, o gasto com ração iria aumentar muito e não compensaria o pequeno incremento no ganho de peso que esse manejo viria a oferecer.

\section{Conclusões}

1. Os juvenis de pirarucu apresentam preferência alimentar pelo período noturno, principalmente no início da noite.

2. O manejo alimentar indicado para o pirarucu, em uma criação intensiva, é a alimentação durante o período diurno.

\section{Agradecimentos}

Ao CNPq e à Agencia Española de Cooperación Internacional (AECI), pelo suporte financeiro.

\section{Referências}

AMUNDSEN, P.A.; GABLER, H.M.; HERFINDAL, T.; RIISE, L.S. Feeding chronology of Atlantic salmon parr in subartic rivers: consistence of nocturnal feeding. Journal of Fish Biology, v.56, p.676-686, 2000.

AZZAYDI, M.; MARTINÉZ, F.J.; ZAMORA, S.; SÁNCHEZVÁZQUEZ, F.J.; MADRID, J.A. Effect of meal size modulation on growth performance and feeding rhythms in European sea bass (Dicentrarchus labrax, L.). Aquaculture, v.170, p.253-266, 1999.

AZZAYDI, M.; MARTINÉZ, F.J.; ZAMORA, S.; SÁNCHEZVÁZQUEZ, F.J.; MADRID, J.A. The influence of nocturnal vs. diurnal feeding under winter conditions on growth and feed conversion of European sea bass (Dicentrarchus labrax, L.). Aquaculture, v.182, p.329-338, 2000.

BARAS, E.; MELARD, C.; GRINARD, J.C.; THOREAU, X. Comparison of food conversion by pirapitinga Piaractus 
brachypomus under different feeding time. Progressive FishCulturist, v.58, p.59-61, 1996.

BOLLIET, V.; CHEEWASEDTHAM, C.; HOULIHAN, D.; GÉLINEAU, A.; BOUJARD, T. Effect of feeding time on digestibility, growth performance and protein metabolism in the rainbow trout Oncorhynchus mykiss: interactions with dietary fat levels. Aquatic Living Resources, v.13, p.107-113, 2000.

BOUJARD, T. Diel rhythms of feeding activity in the European catfish, Silurus glandis. Physiology and Behavior, v.58, p.641645, 1995.

BOUJARD, T.; LEATHERLAND, J.F. Circadian rhythms and feeding time in fishes. Environmental Biology of Fishes, v.35, p.109-131, 1992.

CAVERO, B.A.S.; PEREIRA-FILHO, M.; ROUBACH, R.; ITUASSU, D.R.; GANDRA, A.L.; CRESCÊNCIO, R. Biomassa sustentável de juvenis de pirarucu em tanques-rede de pequeno volume. Pesquisa Agropecuária Brasileira, v.38, p.723-728, 2003.

GANDRA, A.L. Estudo da freqüência alimentar do pirarucu, Arapaima gigas (Cuvier, 1829). 2002. 36p. Dissertação (Mestrado) - Universidade Federal do Amazonas, Manaus.

GARCIA, L.; BARDALES, F. de M.; MORI, L. Influencia de tres tipos de dieta en el crecimiento de alevinos de Paiche (Arapaima gigas, Cuvier 1870). In: CONGRESSO IBEROAMERICANO VIRTUAL DE ACUICULTURA, 1., 2002. [Anais.]. p.518-528. Disponível em: <http://www.civa2002.org>. Acesso em: 24 ago. 2002.

GELINEAU, A.; MAMBRINI, M.; LEATHERLAND, J.F.; BOUJARD, T. Effect of feeding time on hepatic nucleic acid, plasma T3, T4, and GH concentrations in rainbow trout. Physiology and Behavior, v.59, p.1061-1067, 1996.

GREENWOOD, M.F.D.; METCALFE, N.B. Minnows become nocturnal at low temperatures. Journal of Fish Biology, v.53, p.2532, 1998.

HEILMAN, M.J.; SPIELER, R.E. The daily feeding rhythm to demand feeders and the effects of timed meal-feeding on the growth of juvenile Florida pompano, Trachinotus carolinus. Aquaculture, v.180, p.53-64, 1999.

HONCZARYCK, A.; MAEDA, L.S. Crescimento do pirarucu, Arapaima gigas, utilizando dieta à base de ensilado biológico de pescado. In: CONGRESSO SUL-AMERICANO DE AQÜICULTURA, 1., 1998, Recife. Anais. Recife: Simbraq, 1998. p.93-100.

HOSSAIN, M.A.R.; HAYLOR, G.S.; BEVERIDGE, M.C.M. Effect of feeding time on the growth and feed utilization of African catfish Clarias gariepinus (Burchell 1822) fingerlings. Aquaculture Research, v.32, p.999-1004, 2001.

IMBIRIBA, E.P. Potencial de criação de pirarucu, Arapaima gigas, em cativeiro. Acta Amazonica, v.31, p.299-316, 2001.

IMRE, I.; BOISCALAR, D. Age effects on diel activity patterns of juvenile Atlantic salmon: parr are more nocturnal than young-of-theyear. Journal of Fish Biology, v.64, p.1731-1736, 2004.

ITUASSÚ, D.R.; PEREIRA-FILHO, M.; ROUBACH, R.; CRESCÊNCIO, R.; CAVERO, B.A.S.; GANDRA, A.L. Níveis de proteína bruta para juvenis de pirarucu. Pesquisa Agropecuária Brasileira, v.40, p.255-259, 2005.

JOBLING, M.; KOSKELA, J.; PIRHONEN, J. Feeding time, feed intake and growth of baltic salmon, Salmo salar, and brown trout, Salmo trutta, reared in monoculture and duoculture at constant low temperature. Aquaculture, v.163, p.73-84, 1998.

LOPÉZ-VÁSQUEZ, K. Variação circadiana da atividade das enzimas digestivas amilase, maltase, protease e lipase em juvenis de tambaqui Colossoma macropomum Cuvier, 1818. 2001. 66p. Dissertação (Mestrado) - Universidade Federal do Amazonas, Manaus.

ONO, E.A.; HALVERSON, M.R.; KUBITZA, F. Pirarucu o gigante esquecido. Panorama da Aqüicultura, v.14, p.14-25, 2004.

PEREIRA-FILHO, M.; CAVERO, B.A.S.; ROUBACH, R.; ITUASSÚ, D.R.; GANDRA, A.L.; CRESCÊNCIO, R. Cultivo do pirarucu (Arapaima gigas) em viveiro escavado. Acta Amazonica, v.33, p.715-718, 2003.

RUBIO, V.C.; SÁNCHEZ-VÁSQUEZ, F.J.; MADRID, J.A. Nocturnal feeding reduces sea bass (Dicentrarchus labrax) pelletcatching ability. Aquaculture, v.220, p.697-705, 2003.

SMITH, N.J.H. A pesca no Rio Amazonas. Manaus: Inpa, 1979. 154p. 